\title{
Novel diagnostic markers for endometriosis
}

\author{
Żaneta Kimber-Trojnar ${ }^{1 *}$, Aleksandra Pilszyk ${ }^{2}$, Magdalena Niebrzydowska ${ }^{2}$, Zuzanna Pilszyk ${ }^{3}$, Monika Ruszała \\ 1 and Bożena Leszczyńska-Gorzelak ${ }^{1}$

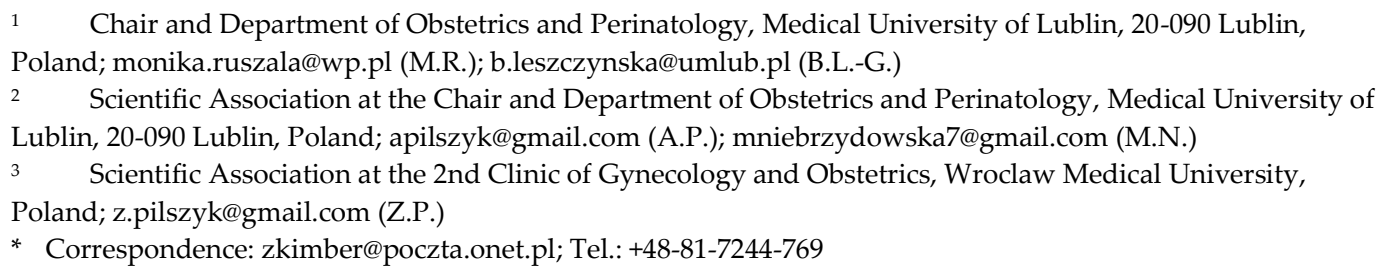

\begin{abstract}
Endometriosis is a disease that affects women of reproductive age and has a significant impact on their well-being. The main symptoms are dysmenorrhea, chronic pelvic pain and infertility. The diagnostic process in many cases is very long and can take up to 8-12 years. Laparoscopy, which is an invasive method, is still necessary to confirm final identification. Therefore, the development of diagnostic markers seems to be crucial for the diagnosis and proper treatment of women affected by endometriosis as soon as possible. Still the most frequently studied and used marker is Cancer Antigen 125 (CA-125). Other glycoproteins, growth factors and immune markers seem to play an important role. However, the search for the ideal endometriosis marker is still ongoing. Developing researches on endometriosis pathogenesis help to identify potential biomarkers or sets of biomarkers in order to improve and speed up the diagnostic process in a non-invasive way.
\end{abstract}

Keywords: endometriosis; diagnostic markers; CA-125; urocortin; activin A; follistatin; microRNA; integrins

\section{Introduction}

Endometriosis is a disease with features of chronic inflammation. It is characterized by the presence of endometrial-like tissue outside the uterus. The most common locations for ectopic endometrial implants are ovaries, peritoneum and rectovaginal septum. [1,2] There are three types of endometriosis: peritoneal, ovarian and deeply infiltrating. [3].

The incidence of endometriosis in women of reproductive age varies between $6-10 \%$. It is also considered that endometriosis occurs in $21-47 \%$ of women with infertility and $71-$ $87 \%$ with chronic pelvic pain. Endometriosis is a major cause compromised quality of life in affected women [2].

The most common symptoms of endometriosis are painful sexual intercourse (deep dyspareunia), pain before and/or during menstruation (dysmenorrhoea), pain with urination (dysuria) and chronic pelvic pain $[2,4]$. The progression of the disease does not correlate with the aggravation of symptoms and none of them is specific. Therefore, the time from the first symptoms to obtaining the diagnosis may last about 8-12 years [4]. As a result of improper verification process patient may be unnecessarily treated for diseases that may mimic the symptoms associated with other chronic pain-related disorders, such as irritable bowel syndrome and pelvic inflammatory disease [5,6]. In addition, women with endometriosis experience a number of non-clinical symptoms that include depression, fatigue, and feeling of isolation. Endometriosis has a negative impact on psychological and social welfare [7].

The gold standard in the diagnosis of endometriosis is still invasive examination laparoscopy, preceded by transvaginal ultrasound and pelvic magnetic resonance imaging (MRI) [6]. It is considered that the development of non-invasive diagnostic tests such 
as 'biomarkers' would have a clear impact in reducing diagnosis time and monitoring the progress of the disease and the effectiveness of treatment [5]. To replace invasive diagnostic methods, biomarkers could be considering clinically useful if they comply with predetermined criteria - sensitivity $94 \%$ and specificity $79 \%[8,9]$.

This paper aims to describe and discuss the current status of biomarkers of endometriosis in serum. In our review we focused on the main groups of markers which are: glycoproteins, growth factors, peptides, immunological markers, markers of oxidative stress and microRNA (Figure 1).

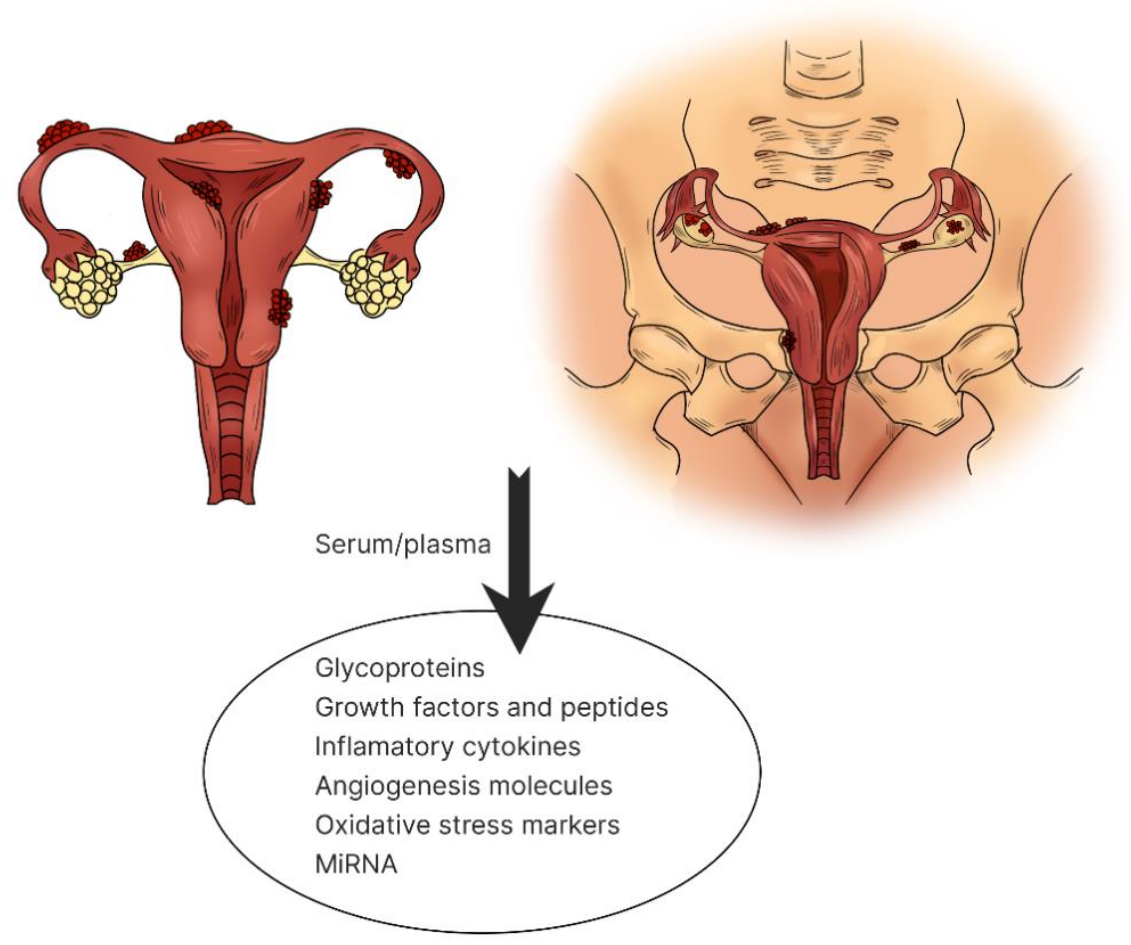

Figure 1. The most typical locations of ectopic endometriosis implants. Several potential endometriosis biomarkers are produced by the endometriosis implants themselves, by affected tissues and/or by the immune system

We conducted a comprehensive literature review using electronic databases such as Pubmed, Science Direct and Google Scholar. The review was limited to sources in English language. We considered articles published until March 2021. Keywords such as : "endometriosis", "glycoproteins", "urocortin", "immunological markers", "oxidative stress", "microRNA" and various combinations of the above were used. Publications were selected if they related to studies conducted on potential biomarkers detected in women with endometriosis. In addition, we manually reviewed the references for each article to find potentially missed studies. Besides, we identified 345 articles that were related to topics of interest. After excluding duplicates, 47 studies were selected for analysis.

\section{Glycoproteins}

Many studies have evaluated the usefulness of serum glycoproteins as diagnostic tools in endometriosis. In medicine they are commonly used for the diagnosis and evaluation of malignant disease [10].

The most commonly described glycoprotein as a potential marker for endometriosis is Cancer Antigen 125 (CA-125). It is a well-known tumor marker of the ovarian epithelial cells, which originates from the coelomic epithelium, including the endometrium, fallopian tube, ovary and peritoneum [11]. 
CA-125 is not a specific marker. Its elevated concentration occurs in patients with cancer of the breast, endometrium, lung, gastrointestinal and inflammatory conditions. Increase of CA-125 represents the most reliable marker for identification of epithelial ovarian cancer. However, its suitability is also tested in endometriosis, which is an inflammatory disease and in the course of this disease CA-125 is secreted into circulation by endometrial and mesothelial cells [12,13].

To date, there is no clearly defined marker limit value. Most articles consider $35 \mathrm{U} / \mathrm{mL}$ as a cut-off point. It is assumed that the level of marker in women before and after menopause is different. It was investigated that before menopause the best cut-off point was 37 $\mathrm{U} / \mathrm{mL}$ and $35 \mathrm{U} / \mathrm{mL}$ in postmenopausal patients [14].

The results of sensitivity for CA-125 vary between different studies [15-17]. Although, CA-125 values fluctuate during the different phases of the menstrual cycle, the value is usually higher during menstruation [12]. This is probably due to the increased inflammatory activity of endometrial cells. It is proposed to test the concentration of CA125 in two phases of the cycle - in the middle of the cycle and in the menstrual phase. Positive results of CA-125 in the middle of the menstrual cycle indicate a very high risk of endometriosis [12].

It has been scientifically proven that there is a correlation between high CA-125 and the stage of the disease and its clinical type [18]. The sensitivity of endometriosis stage III and IV was $63.1 \%$, compared to only $24.8 \%$ in stage I and II. Thus, investigation of concentration of the marker may have a higher value in deeply infiltrating endometriosis with present adhesions [19].

Currently, despite its relatively low sensitivity and specificity, CA-125 remains the only marker widely used in clinical practice in the diagnosis of endometriosis. To date, CA-125 may be suggested as a prognostic rather than diagnostic marker. It is believed that in women with endometriosis symptoms a result above $\geq 35 \mathrm{U} / \mathrm{mL}$ may result in shorter diagnosis times and faster implementation of appropriate therapy $[19,20]$.

CA 19-9 is a tumor marker which has been used especially in the diagnosis of pancreatic cancer and gastrointestinal cancers. When it became clear that endometrium also produces CA 19-9, researchers began to look for its application in diagnosing endometriosis. However, the results of these researches are strongly divided. (8) (21) Some study reported that CA 19-9 is not related to endometriosis [21], while other researches have noted an increase levels of these marker in women with advanced stages of endometriosis [12]. Comparing to CA-125 its specificity and sensitivity are respectively $86-89 \%$ and $52-$ $61 \%$ [22].

Other glycoproteins that were taken into consideration in the studies were CA 15-3, CA 72-4, $\alpha$-fetoprotein (AFP) and carcinoembryonic antigen (CEA). Nevertheless, the results of these research indicate that it is unlikely to have any diagnostic value in case of endometriosis.

\section{Growth factors and peptides: urocortin, activin, follistatin}

Few studies have evaluated the use of urocortin as a diagnostic marker of symptomatic endometriosis.

Urocortin is a member of corticotrophin-releasing hormone $(\mathrm{CRH})$ family and is produced by eutopic and ectopic endometria. It is also believed to play an important role in decidualization, which is essential process during early pregnancy [23]. Another effect of the urocortin is the mediation in the process of mast cell degranulation and increasing the permeability of blood vessels [24]. Three types of urocortin (i.e. Ucn1, Ucn2, Ucn3), which interact with two types of CRH receptor, can be distinguished. Ucn 1 binds type 1 and type 2 CRH receptors, while Ucn2 and Ucn3 bind selectively to CRH-R2 [25].

According to some studies, evaluating plasma urocortin levels can detect symptomatic endometriosis with high sensitivity.

Maia et al. conducted a study in patients suffering from infertility and/or chronic pelvic pain [26]. The purpose of the study was to assess the predictive value of Ucn1 in the detection of endometriosis in women with the above mentioned symptoms. Women 
with symptomatic endometriosis had higher levels of Ucn1 (median $59 \mathrm{pg} / \mathrm{mL}$, interquartile interval 48-107 pg/mL) compared to women with no lesions (median $34 \mathrm{pg} / \mathrm{mL}$, interquartile interval 22-43 pg/mL). Moreover, women with disorders other than endometriosis also had elevated urocortin levels, but to a lesser extent. The foregoing results show that the increase in plasma Ucn $1>46 \mathrm{pg} / \mathrm{mL}$ allows to differentiate the occurrence of endometriosis in women compared to those with no lesions ( $76 \%$ sensitivity, $88 \%$ specificity). However, it is not possible to distinguish endometriosis from other diseases (including ovarian teratoma, ectopic pregnancy, uterine leiomyoma). It is also important to note that the highest detection rate of endometriosis occurred in women who suffered from both infertility and chronic pelvic pain.

It is also suspected that there may be a link between variations in CRH/Ucn1 levels and progesterone resistance, due to lack of growth of Ucn1 and CRH mRNA levels during the secretory phase of the menstrual cycle in women with endometriosis. Novembri et al. demonstrated that the expression of Ucn1 and CRH mRNA in healthy women was higher in the secretory phase compared to the proliferative phase, while in women with endometriosis it was the same in both phases [23].

According to Florio et al. urocortin levels in women with endometriosis was twice as high as in women with non-endometrial ovarian cysts (median $49 \mathrm{pg} / \mathrm{mL}$, interquartile interval $41-63 \mathrm{pg} / \mathrm{mL}$ vs median $19 \mathrm{pg} / \mathrm{mL}$, interquartile interval $15-23 \mathrm{pg} / \mathrm{mL}$ ) and was significantly higher in cystic content of endometriomas compared to peritoneal fluid and plasma [27]. Elevated urocortin level detected endometriosis with $88 \%$ sensitivity and $90 \%$ specificity, while CA- 125 detected only $65 \%$ of cases with the same specificity.

Nevertheless, not all of the studies confirm the usefulness of urocortin as a marker of symptomatic endometriosis [28,29]. In researches comparing the level of urocortin in women with endometriosis and ovarian teratomas and between endometriosis and benign ovarian cysts, no significant differences were observed.

Activin $A$ is a growth factor belonging to the transforming growth factor $\beta$ (TGF- $\beta$ ) family. Physiologically it is produced by a healthy endometrium and its expression reaches peak values in the secretory phase of the menstrual cycle [30]. Activin A promotes the process of decidualization and is also believed to play a role in the immunological processes of cells involved in the pathogenesis of endometriosis. It has been noticed that in endometriosis its level increases both in eutopic and ectopic endometria. The highest increase was observed in ovarian endometrioma (OMA) in comparison with other types of endometriosis, but its growth was insufficient compared to controls to be used as a marker [31].

Follistatin is an extracellular glycoprotein secreted at a constant level throughout the whole menstrual cycle and its growth is observed during early pregnancy. Its main action is the neutralization of activin A, which leads to inhibition of the decidualization process [32]. The highest increase in plasma follistatin level was observed in the OMA and peritoneal forms in relation to deep infiltrating endometriosis (DIE) and healthy controls, which excludes its use as a marker of endometriosis [31].

The combination of activin A and follistatin as markers of endometriosis showed the highest effectiveness. In this case, a significant increase in the form of OMA was observed, but it was not suitable to differentiate the other forms of endometriosis from healthy controls [31].

\section{Immunological markers}

There are many indications that the dysfunction of the immune system is involved in the pathogenesis of endometriosis. Many studies have been conducted to determine whether different populations of immune cells could be used as non-invasive markers of endometriosis.

Macrophages are one of the cells found in significant amounts in the peritoneal fluid. They are responsible for ectopic endometrial cell adhesion, implantation and growth. What is more, macrophages secrete numerous substances which are said to influence the development of endometriosis [33]. 
Macrophages are considered to be the source of vascular endothelial growth factors (VEGF) in women with endometriosis. VEGF is responsible for angiogenesis in the endometrial tissue, which allows it to regenerate after menstruation, but also affects newly formed vessels. Mouse studies showed that after implantation of uterine tissue into the peritoneum, macrophages activation and increased VEGF secretion in response to tumor necrosis factor $\alpha$ (TNF- $\alpha$ ) and interleukin 6 (IL-6) occurred [34]. According to some study the level of TNF- $\alpha$ increased in patients with endometriosis and correlated with its severity [35].

Studies on macrophage migration inhibitory factor (MIF) have shown that it is a cytokine with strong immunoregulatory potential, affecting angiogenesis and tissue remodeling [36]. It has been observed to significantly increase in endometrial lesions, especially in advanced stages of the disease [37].

Natural Killer (NK) cells may play an important role in the pathogenesis of endometriosis. It is believed that they are responsible for clearance of regurgitated endometrial cells from the peritoneal cavity. It has been observed that patients with endometriosis have reduced NK cell cytotoxicity. This suggests that NK cell dysfunction may allow implantation of endometrial cells into the peritoneal cavity and lead to endometriosis [38]. IL-12 may inhibit process of endometriosis by activation of NK cells [39]. It has also been demonstrated that abnormal human leukocyte antigen (HLA) class I and II expression leads to a decrease in their cytotoxic activity [40].

A compound such as soluble intercellular-adhesion molecule-1 (sICAM-1) should also be distinguished. It is associated with reduced cytotoxic activity of NK cells. It is believed that it may be relevant to implantation disorders and the formation of endometrial lesions [33]. Matalliotakis et al. demonstrated that the level of sICAM-1 was higher in women suffering from endometriosis infertility compared to healthy controls [41].

Additionally, elevated monocyte chemotactic protein-1 (MCP-1) values were observed in peritoneal fluid and plasma in women with endometriosis, especially in the early stages of the disease. Another study revealed its elevated values in the more advanced stages [8].

According to Cho et al. the use of neutrophil/lymphocyte ratio can be applied as a diagnostic method for endometriosis [42]. They have shown that women with endometriosis may have neutrophilia coexisting with lymphocytopenia. The combined use of neutrophil/lymphocyte ratio and CA-125 concentration demonstrated high sensitivity for endometriosis detection with sensitivity of $69.3 \%$ and specificity of $83.9 \%$ [42].

\section{Oxidative stress}

The formation of reactive oxygen species (ROS) is physiological process regulated by antioxidant defense mechanisms. Imbalance between these two formations is called oxidative stress. Its significant role was demonstrated in inflammatory response of many diseases including endometriosis [9].

Inadequate metabolism of free radicals and ROS has a significant impact on the use of thiols and carbonyls, which seem to be associated with endometriosis and subfertility. According to one study, the amount of these substances is significantly reduced in the presence of endometriosis compared to controls. However, other studies have shown that there is no link between endometriosis and the presence of oxidative stress markers [43].

Due to the multitude of factors regulating the level of oxidative stress, authors highlight that there is need of further studies to conclude if there is possible use of oxidative stress markers as diagnostic tests for endometriosis [9,44].

\section{MicroRNAs}

MicroRNA (miRNA) is a small non-coding RNA molecule, containing about 22-24 nucleotides. Its main function is regulation of gene expression, it also affects processes of proliferation, differentiation, growth, and apoptosis. MiRNAs are regulatory molecules that control the expression of many genes and play key roles in many biological processes 
[45]. In turn, dysregulation of microRNA has been associated with many diseases including endometriosis. MicroRNA became new perspective in field of serum markers and has become the subject of many research papers [46,47].

In 2020, Zhang et al. selected and tested specific types of miRNA: miR-134-5p, miR197-5p, miR-22-3p, miR-320a, miR-494-3p, and miR-939-5p [47]. Two types of miRNA: miR-22-3p and miR-320a, were distinctly upregulated in group of endometriosis patients in comparison to the reference group. Also the distinctive difference was noticed between patients in stage I-II compared to stage III-IV. It seems that these two types of miRNA could be potential biomarkers for endometriosis. [47].

MiRNAs are very attractive diagnostic markers due to their lower complexity, tissue specificity, lack of known post-translational modifications and stability in blood, urine or tissues [48]. Researchers summed up studies about miRNA as endometriosis marker and concluded that the average value of sensitivity was $86 \%$ and specificity was $88 \%$ [49]. Although the results are promising authors note that their assessment may be affected by menstruation cycle. Also miRNA research remains new field of study and requires confirmation and further investigation $[47,49]$.

\section{Integrins as cell-adhesion molecules}

Integrins are the main protein receptors, also known as transmembrane linkers, which participate both in binding cells and transferring signals from and to extracellular matrix. They are involved in the regulation of pathophysiological processes such as cell adhesion, proliferation and migration [50].

Depolarized integrin $\alpha 6$ was examined as a possible marker of endometriosis. Researchers evaluated the percentage of positive glandular cells and the location of expression in each sample section, after a previous biopsy. An immunohistochemical (IHC) method was used. Integrin $\alpha 6$ was considered to be polarized when expression was shown only on the basal side of the cell and depolarized when expression was observed on any side of the cell. Using depolarized expression as the positive test result, the sensitivity was $67 \%$ and the specificity was $84 \%$. Such a result does not meet the criteria for either replacement or triage test [51].

Integrin $\beta 1$ is expressed in ectopic endometrial tissues, what may indicate that it is involved in the occurrence of endometriosis. After testing, the following results were obtained: in glandular epithelium, integrin $\beta 1$ had a sensitivity of $18 \%$ and specificity $87 \%$, and in stromal epithelium it had a sensitivity of $76 \%$ and specificity of $0 \%[51,52]$.

Researchers attempted to determine the expression of $\alpha 3 \beta 1$ and $\alpha 4 \beta 1$ integrins in endometrial biopsy samples from women with endometriosis. In glandular epithelium, $\alpha 3 \beta 1$ integrin demonstrated a sensitivity of $100 \%$ and a specificity of $27 \%$, and in stromal epithelium, it had a sensitivity of $53 \%$ and a specificity of $27 \%$. In turn, $\alpha 4 \beta 1$ integrin in glandular epithelium had a sensitivity of $65 \%$ and specificity of $40 \%$, and in stromal epithelium it demonstrated a sensitivity of $59 \%$ and a specificity of $20 \%[51,53]$.

In conclusion, the results for all the above integrins as biomarkers for endometriosis were discouraging. They are not sufficient to draw conclusions regarding their role in detecting endometriosis.

\section{Conclusions}

Due to endometriosis heterogeneity, perfect diagnostic markers should give on array of results fulfilling the requirements of specificity and sensitivity at the same time. The broad implication of the present research showed that even though we have a number of promising markers none of them meets the mentioned criteria on its own [Table 1].

Table 1. Possible diagnostic markers for endometriosis 


\begin{tabular}{|c|c|c|c|c|c|}
\hline Glycoproteins & Growth factors & $\begin{array}{c}\text { Immunological } \\
\text { markers }\end{array}$ & Oxidative stress & mRNA & $\begin{array}{c}\text { Cell-adhesion } \\
\text { molecules }\end{array}$ \\
\hline CA-125 & Urocortin & VEGF & & miR-134-5p & integrins $\alpha 3 \beta 1$ \\
\hline CA 19.9 & Activin A & TNF- & & miR-197-5p & integrins $\alpha 4 \beta 1$ \\
\hline \multirow[t]{2}{*}{ CA 15.3} & Follistatin & IL-6 & & miR-22-3p & integrins $\beta 1$ \\
\hline & & & ROS & & \\
\hline CA 72 & & NK & & miR-320a & integrins $\alpha 6$ \\
\hline $\mathrm{AFP}$ & & slCAM-1 & & miR-494-3p & \\
\hline CEA & & MCP-1 & & miR-939-5p & \\
\hline
\end{tabular}

Further studies are certainly required to combine all of available methods, including serum markers, glycoproteins, growth factors and peptides, immunological markers also genomic technologies and non-invasive imaging methods such as ultrasonography (USG) or MRI. A variety of combined tests should help to unify the results and as an effect lead to faster diagnosis what will have a direct impact on patients quality of life.

Author Contributions: Conceptualization, A.P., M.N. and Z.P.; resources, A.P., M.N. and Z.P.; writing-original draft preparation, A.P., M.N., Z.P., M.R. and Z.K.T.; writing-review and editing, A.P., M.N., Z.P., M.R. and Ż.K.T.; visualization, A.P., M.N. and Z.P.; supervision, Ż.K.-T. and B.L.G.; funding acquisition, $\dot{Z}$. K.-T. All authors have read and agreed to the published version of the manuscript.

Funding: This research received no external funding.

Institutional Review Board Statement: Not applicable.

Data Availability Statement: MDPI Research Data Policies.

Conflicts of Interest: The authors declare no conflict of interest.

\section{References}

1. Hickey, M.; Ballard, K.; Farquhar, C. Endometriosis. BMJ 2014, 348, 29-34.

2. Falcone, T.; Flyckt, R. Clinical Management of Endometriosis. Obstet. Gynecol. 2018, 131, 557-571.

3. Basta, A.; Brucka, A.; Górski, J.; Kotarski, J.; Kulig, B.; Oszukowski, P.; Poreba, R.; Radowicki, S.; Radwan, J.; Sikora, J.; Skret, A.; Skrzypczak, J.; Szyłło, K. The statement of Polish Society's Experts Group concerning diagnostics and methods of endometriosis treatment. Ginekol. Pol. 2012, 83, 871-876.

4. Giudice, L.C. Clinical practice. Endometriosis. N. Engl. J. Med. 2010, 362, 2389-2398.

5. May, K.E.; Conduit-Hulbert, S.A.; Villar, J.; Kirtley, S.; Kennedy, S.H.; Becker, C.M. Peripheral biomarkers of endometriosis: a systematic review. Hum. Reprod. Update 2010, 16, 651-74.

6. Kiesel, L.; Sourouni, M. Diagnosis of endometriosis in the 21st century.Climacteric 2019, 22, 296-302.

7. Bourdel, N.; Chauvet, P.; Billone, V.; Douridas, G.; Fauconnier, A.; Gerbaud, L.; Canis, M. Systematic review of quality of life measures in patients with endometriosis. PLOS ONE 2019, 14, e0208464.

8. Luisi, S.; Pinzauti, S.; Regini, C.; Petraglia, F. Serum markers for the noninvasive diagnosis of endometriosis. Womens Health (Lond.) 2015, 11, 603-610.

9. Coutinho, L.M.; Ferreira, M.C.; Rocha, A.L.L.; Carneiro, M.M.; Reis, F.M. New biomarkers in endometriosis. Adv. Clin. Chem. 2019, 89, 59-77.

10. Fassbender, A.; Burney, R.O.; O, D.F.; D'Hooghe, T.; Giudice, L. Update on Biomarkers for the Detection of Endometriosis. Biomed. Res. Int. 2015, 2015, 130854.

11. Jacobs, I. Screening for ovarian cancer by CA-125 measurement. Lancet 1988, 16;1(8590), 889.

12. Oliveira, M.A.P.; Raymundo, T.S.; Soares, L.C.; Pereira, T.R.D.; Demôro A.V.E. How to Use CA-125 More Effectively in the Diagnosis of Deep Endometriosis. Biomed. Res. Int. 2017, 2017, 9857196. 
13. Mol, B.W.J.; Bayram, N.; Lijmer, J.G.; Wiegerinck, M.A.; Bongers, M.Y.; van der Veen, F.; Bossuyt, P.M. The performance of CA125 measurement in the detection of endometriosis: a meta-analysis. Fertil. Steril. 1998, 70, 1101-1108.

14. Karimi-Zarchi, M.; Dehshiri-Zadeh, N.; Sekhavat, L.; Nosouhi, F. Correlation of CA-125 serum level and clinico-pathological characteristic of patients with endometriosis. Int. J. Reprod. Biomed. (Yazd) 2016, 14, 713-718.

15. Moretuzzo, R.W.; DiLauro, S.; Jenison, E.; Chen, S.L.; Reindollar, R.H.; McDonough, P.G. Serum and peritoneal lavage fluid CA-125 levels in endometriosis. Fertil. Steril. 1994, 61, 438-442.

16. Franchi, M.; Beretta, P.; Zanaboni, F.; Donadello, N.; Ghezzi, F. Use of serum CA125 measurement in patients with endometriosis. Italian J. Gynaecol. Obstet. 1993, 5, 149-153.

17. Hirsch, M.; Duffy, J.M.; Deguara, C.S.; Davis, C.J.; Khan, K.S. Diagnostic accuracy of Cancer Antigen 125 (CA125) for endometriosis in symptomatic women: a multi-center study. Eur. J. Obstet. Gynecol. Reprod. Biol. 2016, 210, $102-107$.

18. Tian, Z.; Chang, X.H.; Zhao, Y.; Zhu, H.L. Current biomarkers for the detection of endometriosis. Chin. Med. J. 2020, 133, 23462352.

19. Hirsch, M.; Duffy, J.M.N.; Davis, C. J.; Nieves Plana, M.; Khan, K.S. Diagnostic accuracy of cancer antigen 125 for endometriosis: a systematic review and meta - analysis. BJOG 2016, 123, 1761-1768.

20. Kafali, H.; Artuc, H.; Demir, N. Use of CA-125 fluctuation during the menstrual cycle as a tool in the clinical diagnosis of endometriosis. J. Obstet. Gynecol. Reprod. Biol. 2004, 116, 85-88.

21. Somigliana, E.; Viganö, P.; Tirelli, A.S. Use of the concomitant serum dosage of CA-125, CA 19-19 and interleukin-6 to detect the presence of endometriosis. Results from a series of reproductive age women undergoing laparoscopic surgery for benign gynaecological conditions. Hum. Reprod. 2004, 19, 1871-1876.

22. Kurdoglu, Z.; Gursoy, R.; Kurdoglu, M.; Erdem, M.; Erdem, O.; Erdem, A. Comparison of the clinical value of CA 19-9 versus CA 125 for the diagnosis of endometriosis. Fertil. Steril. 2009, 92, 1761-1763.

23. Novembri, R.; Borges, L.E.; Carrarelli, P.; Rocha, A.L.; De Pascalis, F.; Florio, P.; Petraglia, F. Impaired CRH and urocortin expression and function in eutopic endometrium of women with endometriosis. J. Clin. Endocrinol. Metab. 2011, 96, 1145-1450.

24. Yalcin, S.E.; Ocal, I.; Yalcin, Y.; Selim, H.S.; Caltekin, M.D.; Aydogmus, H.; Kelekci, S. Evaluation of the Ki-67 proliferation index and urocortin expression in women with ovarian endometriomas. Eurasian J. Med. 2017, 49, 107-112.

25. Liew, H.K.; Huang, L.C.; Yang, H.I.; Peng, H.F.; Li, K.W.; Tsai, A.P.; Chen, S. Y.; Kuo, J.S.; Pang C.Y. Therapeutic effects of human urocortin-1, -2 and -3 in intracerebral hemorrhage of rats. Neuropeptides 2015, 52, 89-96.

26. Maia, L.M.; Rocha, A.L.; Del Puerto, H.L.; Petraglia, F.; Reis, F.M. Plasma urocortin-1 as a preoperative marker of endometriosis in symptomatisc women. Gynecol. Endocrinol. 2018, 34, 202-205.

27. Florio, P.; Reis, F.M.; Torres, P.B.; Calonaci, F.; Toti, P.; Bocchi, C;. Linton, E.A.; Petraglia, F. Plasma urocortin levels in the diagnosis of ovarian endometriosis. Obstet. Gynecol. 2007, 110, 594-600.

28. Chmaj-Wierzchowska, K.; Kampioni, M.; Wilczak, M.; Sajdak, S.; Opala, T. Novel markers in the diagnostics of endometriomas: Urocortin, ghrelin, and leptin or leukocytes, fibrinogen, and CA-125? Taiwan. J. Obstet. Gynecol. 2015, 54, 126-130.

29. Tokmak, A.; Ugur, M.; Tonguc, E.; Var, T.; Moraloğlu, O.; Ozaksit, G. The value of urocortin and Ca-125 in the diagnosis of endometrioma. Arch. Gynecol. Obstet. 2011, 283, 1075-1079.

30. Rocha, A.L.; Carrarelli, P.; Novembri, R.; Sabbioni, L.; Luisi, S.; Reis, F.M.; Petraglia, F. Altered expression of activin, cripto, and follistatin in the endometrium of women with endometrioma. Fertil. Steril. 2011, 95, 2241-2246.

31. Reis, F.M.; Luisi, S.; Abrão, M.S.; Rocha, A.L.; Viganò, P.; Rezende, C.P.; Florio, P.; Petraglia, F. Diagnostic value of serum activin A and follistatin levels in women with peritoneal, ovarian and deep infiltrating endometriosis. Hum. Reprod. 2012, 27, 14451450 .

32. Torres, P.B.; Florio, P.; Ferreira, M.C.; Torricelli, M.; Reis, F.M.; Petraglia, F. Deranged expression of follistatin and follistatinlike protein in women with ovarian endometriosis. Fertil. Steril. 2007, 88, 200-205.

33. Aznaurova, Y.B.; Zhumataev, M.B.; Roberts, T.K.; Aliper, A.M.; Zhavoronkov, A.A. Molecular aspects of development and regulation of endometriosis. Reprod. Biol. Endocrino. 2014, 12, 50.

34. Lin, Y.J.; Lai, M.D.; Lei, H.Y.; Wing, L.Y. Neutrophils and macrophages promote angiogenesis in the early stage of endometriosis in a mouse model. Endocrinology 2006, 147, 1278-1286.

35. Xavier, P.; Belo, L.; Beires, J.; Rebelo, I.; Martinez-de-Oliveira, J.; Lunet, N.; Barros, H. Serum levels of VEGF and TNF-alpha and their association with C-reactive protein in patients with endometriosis. Arch. Gynecol. Obstet. 2006, 273, $227-231$.

36. Herrmann Lavoie, C.; Fraser, D.; Therriault, M.J.; Akoum, A. Interleukin-1 Stimulates Macrophage Migration Inhibitory Factor Secretion in Ectopic Endometrial Cells of Women with Endometriosis. Am. J. Reprod. Immunol. 2007, 58, 505-513.

37. Morin, M.; Bellehumeur, C.; Therriault, M.J.; Metz, C.; Maheux, R.; Akoum, A. Elevated levels of macrophage migration inhibitory factor in the peripheral blood of women with endometriosis. Fertil. Steril. 2005, 83, 865-872.

38. Eisenberg, V.H.; Zolti, M.; Soriano, D. Is there an association between autoimmunity and endometriosis? Autoimmun. Rev. 2012, 11, 806-814.

39. Itoh, H.; Sashihara, T.; Hosono, A.; Kaminogawa, S.; Uchida, M. Interleukin-12 inhibits development of ectopic endometriotic tissues in peritoneal cavity via activation of NK cells in a murine endometriosis model. Cytotechnology 2011, 63, 133-141.

40. Matalliotakis, I.M.; Goumenou, A.G.; Koumantakis, G.E.; Athanassakis, I.; Dionyssopoulou, E.; Neonaki, M.A.; Vassiliadis, S. Expression of Serum Human Leukocyte Antigen and Growth Factor Levels in a Greek Family With Familial Endometriosis. J. Soc. Gynecol. Investig. 2003, 3, 81-89.

41. Matalliotakis, I.M.; Vassiliadis, S.; Goumenou, A.G.; Athanassakis, I.; Koumantakis, G.E.; Neonaki, M.A.; Koumantakis, E.E. Soluble ICAM-1 levels in the serum of endometriotic patients appear to be independent of medical treatment. J. Reprod. Immunol. 2001, 51, 9-19. 
42. Cho, S.; Cho, H.; Nam, A.; Kim, H.Y.; Choi, Y.S.; Park, K.H.; Cho, D.J.; Lee, B.S. Neutrophil-to-lymphocyte ratio as an adjunct to CA-125 for the diagnosis of endometriosis. Fertil. Steril. 2008, 90, 2073-2079.

43. Hirsch, M.; Davis, C.J.; Preoperative assessment and diagnosis of endometriosis: are we any closer? Curr. Opin. Obstet. Gynecol. 2015, 27, 284-290.

44. Thézénas, M.L.; De Leo, B.; Laux-Biehlmann, A.; Bafligil, C.; Elger, B.; Tapmeier, T.; Morten, K.; Rahmioglu, N.; Dakin, S.G.; Charles, P.; Martinez, F.E.; Steers, G.; Fischer, O.M.; Mueller, J. Amine oxidase 3 is a novel pro-inflammatory marker of oxidative stress in peritoneal endometriosis lesions. Sci. Rep. 2020, 10, 56-65.

45. Wei, Sh.; Xu, H.; Kuang, Y. Systematic enrichment analysis of microRNA expression profiling studies in endometriosis. Iran J. Basic Med. Sci. 2015, 18, 423-429.

46. Goulielmosa, G.N.; Matalliotakisa, M.; Matalliotaki, C.; Eliopoulos, E.; Matalliotakis, I.; Zervou, M.I. Endometriosis research in the -omics era. Gene 2020, 741, 144545.

47. Zhang, L.; Li, H.; Yuan, M.; Li, D.; Sun, C.; Wang, G. Serum Exosomal MicroRNAs as Potential Circulating. Dis. Markers 2020, 2020, 2456340.

48. Moga, M.A.; Bălan, A.; Dimienescu, O.G.; Burtea, V.; Dragomir, R.M.; Anastasiu, C.V. Circulating miRNAs as Biomarkers for Endometriosis and Endometriosis-Related Ovarian Cancer-An Overview. J. Clin. Med. 2019, 8, 735.

49. Zhou, L.; Chen, Y.; Gao, J.; Shankar, S.; Zhang, G. Diagnostic Value of Circulating MicroRNAs for Endometriosis: a Metaanalysis. Reprod. Sci. 2020, 27, 793-805.

50. Schutt, A.K.; Atkins, K.A.; Slack-Davis J.K.; Stovall, D.W.; VCAM-1 on peritoneum and $\alpha 4 \beta 1$ integrin in endometrium and their implications in endometriosis. Int. J. Gynecol. Pathol. 2015, 34, 85-89.

51. Gupta, D.; Hull, M.L.; Fraser, I.; Miller, L.; Bossuyt, P.M.M.; Johnson, N.; Nisenblat, V. Endometrial biomarkers for the noninvasive diagnosis of endometriosis. Cochrane Database Syst. Rev. 2016, 4, CD012165.

52. Duan, R.; Wang, Y.; Lin, A.; Lian, L.; Cao, H.; Gu, W.; Li, T.; Sun, Q. Expression of nm23-H1, p53, and integrin $\beta 1$ in endometriosis and their clinical significance. Int. J. Clin. Exp. Pathol. 2020, 13, 1024-1029.

53. Szymanowski, K.; Skrzypczak, J.; Mikołajczyk, M. Integrin pattern in human endometrium--new diagnostic tool in pelvic endometriosis? Ginekol. Pol. 2003, 74, 257-261. 